() М.О. Корчагина ${ }^{1 *}$, А.А. Трухин ${ }^{2}$, Н.Ю. Свириденко ${ }^{2}$

1Первый Московский государственный медицинский университет им. И.М. Сеченова (Сеченовский Университет), Москва, Россия

${ }^{2}$ Национальный медицинский исследовательский центр эндокринологии, Москва, Россия

В настоящее время синдром Кушинга и его проявления хорошо изучены. При этом основными симптомами гиперкортицизма остаются ожирение, остеопороз, кардиомиопатия, атрофия мышц, истончение кожного покрова и образование багровых полос (стрий) на теле.

На практике самые частые из них - ожирение и остеопороз - встречаются в 90\% случаев. Однако есть ряд пациентов с неявной клинической картиной гиперкортицизма. Часть изменений, наблюдаемых у них, затрагивает глаза. В данной обзорной статье рассматривается редкий симптом гиперкортицизма - экзофтальм. Экзофтальм - это патологическое смещение глазного яблока вперед. Этот симптом известен нам в рамках эндокринной орбитопатии, развивающейся при аутоиммунной патологии щитовидной железы. В статье проводится сравнение механизмов развития глазных симптомов при синдроме Кушинга и болезнях щитовидной железы, в особенности при болезни Грейвса. Обсуждаются возможные молекулярные механизмы, приводящие к экзофтальму у пациентов с синдромом Кушинга. Изучаются факторы, влияющие на адипогенез in vitro и in vivo, в частности факторы, приводящие к увеличению жировой клетчатки орбит на фоне повышенного уровня кортизола. Представлены гормональные сигнальные и транскрипционные каскады, отвечающие за дифференцировку адипоцитов в зрелые жировые клетки. Также в статье рассмотрены другие орбитальные проявления гиперкортицизма, встречающиеся на практике относительно редко. К ним относятся глаукома, а также катаракта, узелки Лиша и центральная серозная хориоретинопатия. Рассмотрены клинические случаи синдрома Кушинга с различными глазными проявлениями и сделаны соответствующие выводы.

КЛЮЧЕВЫЕ СЛОВА: синдром Кушинга; гиперкортицизм; экзофтальм; адипогенез.

\title{
ORBITAL MANIFESTATIONS OF HYPERCORTICISM
}

(c) Maria O. Korchagina ${ }^{1 *}$, Alexey A. Trukhin², Natalya Yu. Sviridenko²

'I.M. Sechenov First Moscow State Medical University (Sechenov University), Moscow, Russia

${ }^{2}$ Endocrinology Research Centre, Moscow, Russia

Nowadays, Cushing's syndrome (hypercortisolism) and its manifestations are well studied. The main symptoms of hypercortisolism are obesity, osteoporosis, cardiomyopathy, muscle atrophy, skin thinning and purple stretch marks (striae) on the body. In practice, obesity and osteoporosis are the most frequent symptoms that are found in $90 \%$ of cases. However, there are some patients with an implicit clinical picture of hypercorticism. Some cases might concomitant with exophthalmos. This review describes a rare symptom of hypercortisolism - exophthalmos. Exophthalmos is a pathological protruding of eyeballs. This symptom is known in the context of TED that occurs most commonly in patients with Graves' disease. The article compares the mechanisms of development of eye symptoms in Cushing's syndrome and thyroid diseases, especially the Graves' disease. It discusses possible molecular mechanisms leading to exophthalmia in patients with Cushing's syndrome. Factors affecting adipogenesis in vitro and in vivo are studied, in particular factors leading to an increase of orbital fatty tissue against of elevated cortisol levels. Hormonal signaling and transcription cascades responsible for adipocyte differentiation into mature fat cells are presented. Other orbital manifestations of hypercortisolism, which occur relatively rare in practice, are also discussed in the article. These include glaucoma as well as cataract, Lisha nodules and central serous chorioretinopathy. Clinical cases of Cushing's syndrome with different ocular manifestations are considered and appropriate conclusions have been drawn.

KEYWORDS: Cushing's syndrome; hypercortisolism; exophthalmos; adipogenesis. 


\section{ВВЕДЕНИЕ}

Гиперкортицизм - это синдром, обусловленный эндогенной гиперпродукцией кортизола или длительным приемом препаратов кортикостероидов. Болезнь Иценко-Кушинга - наиболее частая причина (6070\%) эндогенного гиперкортицизма. В 20-30\% случаев заболевание развивается из-за первичной патологии надпочечников (кортикостерома, первичная двусторонняя макронодулярная гиперплазия при мутациях В гене $A R M(5)$. В 5-10\% случаев адренокортикотропный гормон (АКТГ) синтезирует карциноидная опухоль внегипофизарной локализации (медуллярный рак щитовидной железы, рак клеток островков Лангерганса, хромаффинома, рак яичников, яичек, предстательной железы; карциноид легких, бронхов, тимуса, аппендикса, опухоли желудочно-кишечного тракта, мочевого пузыря, околоушных и слюнных желез) [1]. Распространенность болезни Иценко-Кушинга составляет 39,1 случая на 1 млн, а заболеваемость - 1,2-2,4 случая на 1 млн [2, 3]. В РФ на 2020 г. зарегистрировано 448 пациентов с гиперкортицизмом [4]. Есть ряд исследований, доказывающих более высокую распространенность заболевания в группах потенциального риска (пациенты с ожирением, артериальной гипертензией (АГ), сахарным диабетом (СД) 2 типа) [1, 3]. Кроме того, во всем мире отмечается значительное увеличение числа пациентов с гиперкортицизмом (до 3,9 случая на 1 млн).

Основные проявления заболевания - ожирение, атрофия мышц, истончение кожного покрова и появление стрий из-за распада коллагена и отложения жировой клетчатки в области талии, остеопороз, сопровождаемый компрессионными переломами, кардиомиопатия, стероидный СД, аменорея/эректильная дисфункция и гирсутизм. Также синдром Кушинга связан с целым рядом психических и когнитивных проблем. Большинство пациентов, страдающих синдромом

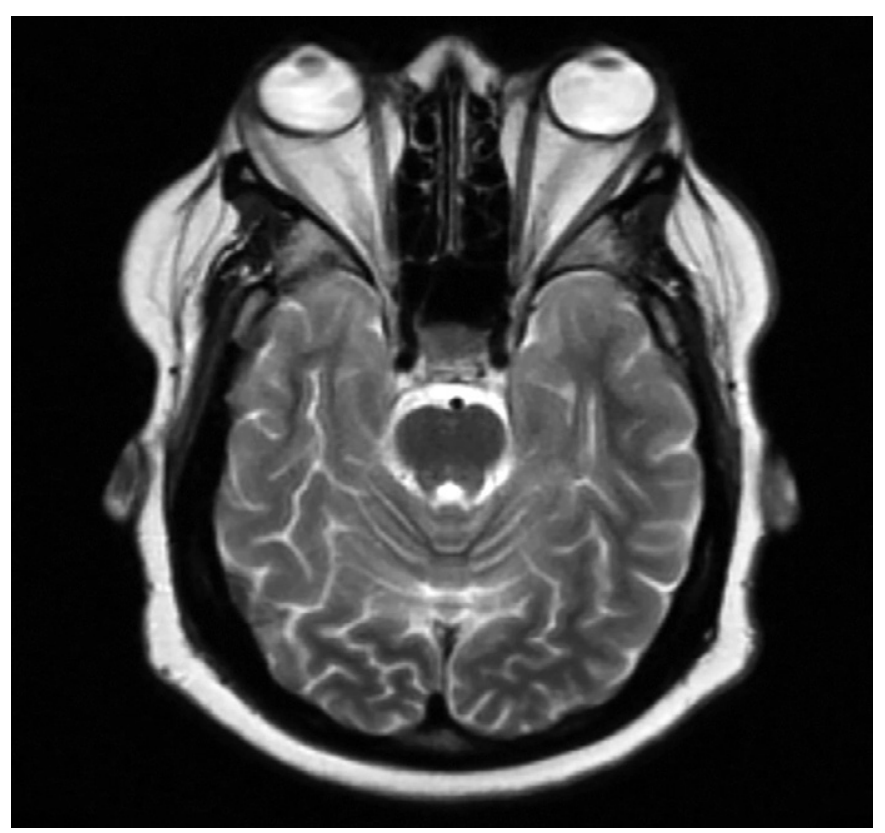

Рисунок 1. Билатеральный экзофтальм по данным магнитнорезонансной томографии у пациента с синдромом Кушинга [5].
Кушинга, соответствуют критериям депрессии, а некоторые пациенты имеют и другие проявления, включая манию, чувство тревоги [3]. Могут развиваться психозы, а у пациентов с эндогенным гиперкортицизмом часто отмечаются суицидальные наклонности. У детей с синдромом Кушинга часто наблюдается обсессивно-компульсивное поведение.

Менее распространенный и недооцененный клинический признак затрагивает глаза. На практике поражение орбит встречается лишь у 30-45\% пациентов с синдромом Кушинга и в ряде случаев даже служит основанием для поиска поражения щитовидной железы и ассоциированной с ним офтальмопатии.

Чтобы описать глазные симптомы, наблюдаемые при болезни Грейвса и синдроме Кушинга, используются разные термины. Термин «эндокринная офтальмопатия» (ЭОП) употребляется в настоящее время как основной для обозначения поражения тканей орбиты при заболеваниях щитовидной железы.

Термином «экзофтальм» обозначают патологическое смещение глазного яблока вперед. Экзофтальм возникает при аутоиммунном поражении тканей орбиты и является одним из клинических проявлений ЭОП. При синдроме Кушинга экзофтальм является отдельным симптомом, возникающим вторично в условиях гиперкортизолемии (рис. 1).

\section{ИЗМЕНЕНИЯ ТКАНИ ОРБИТ ПРИ БОЛЕЗНИ ГРЕЙВСА}

По современным представлениям, ЭОП - это аутоиммунное заболевание, тесно связанное с аутоиммунной патологией щитовидной железы [6]. Предполагается, что щитовидная железа и орбитальные ткани имеют одинаковые антигены, и ЭОП может развиться вследствие перекрестного реагирования антител к антигенам щитовидной железы с тканями орбиты. Экспрессия рецептора тиреотропного гормона (рТТГ) в орбитах может играть ключевую роль в развитии аутоиммунных реакций в орбитальных тканях. Установлено, что у больных с болезнью Грейвса и ЭОП имеется более высокий уровень антител к рТТГ по сравнению с больными болезнью Грейвса без офтальмопатии. Помимо рТТГ существуют и другие кандидаты на рассмотрение в качестве возможных аутоантигенов. Одним из таких кандидатов является рецептор инсулиноподобного фактора роста 1 (рИРФ-1). Он экспрессируется в орбите и в щитовидной железе. Показано, что его уровень коррелирует с активностью и тяжестью ЭОП. In vitro антитела к рИРФ-1, как и антитела к рТТГ, стимулируют дифференцировку орбитальных преадипоцитов в зрелые адипоциты, приводя к увеличению объема ретробульбарной клетчатки (РБК). Симптомы ЭОП чрезвычайно разнообразны. Известно, что экстраокулярные мышцы (ЭОМ) и РБК могут вовлекаться в процесс асимметрично. Увеличение ЭОМ и (или) РБК может приводить к возникновению экзофтальма (проптоза). Установить причину экзофтальма можно с помощью визуализирующих методов исследования - КТ или МРТ. Вовлечение в процесс ЭОМ обнаруживается у большинства пациентов при проведении КТ или МРТ. Увеличение объема РБК при нормальных размерах мышц или их незначительном увеличении 

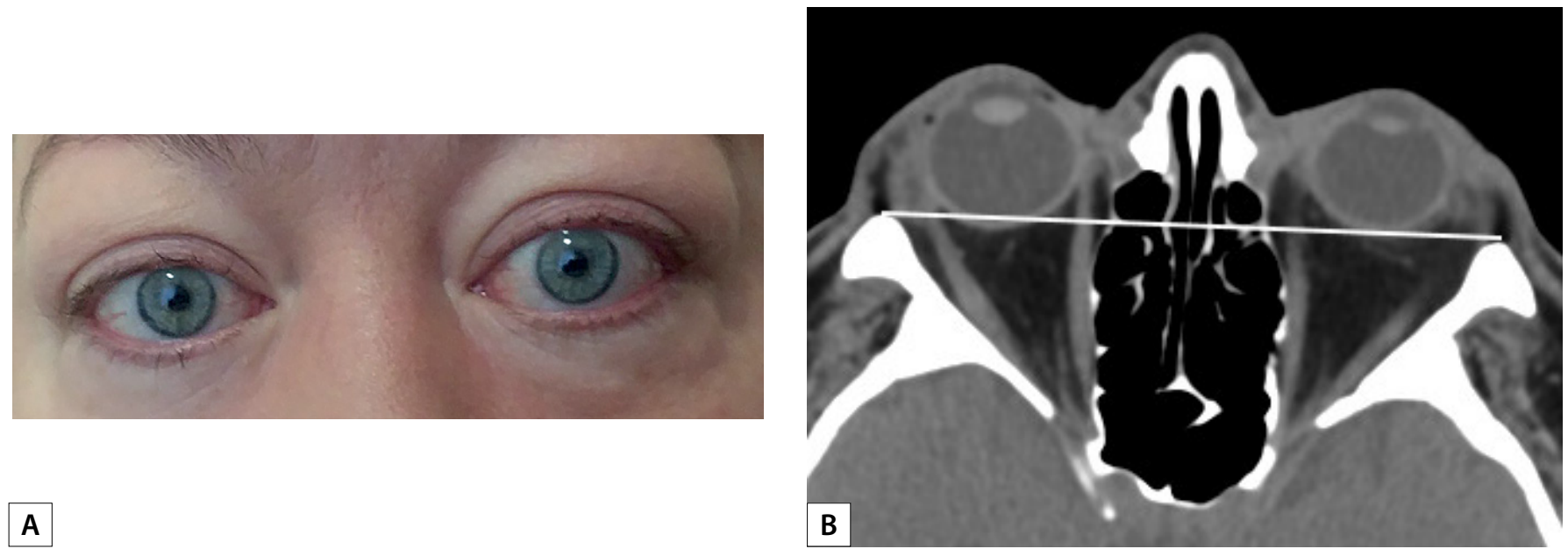
Рисунок 2. «Липогенный» вариант эндокринной офтальмопатии: (А) фото пациента с экзофтальмом; (B) мультиспиральная компьютерная
томография орбит, аксиальная проекция (задний полюс глазного яблока расположен на межскуловой линии - белая линия).

наблюдается значительно реже. Это так называемый «липогенный» вариант ЭОП (рис. 2).

Основной характеристикой «липогенного» варианта является отсутствие увеличения ЭОМ при выраженной пролиферации РБК. Он протекает более доброкачественно и не приводит к ухудшению остроты зрения, кроме того, отсутствуют такие симптомы активности воспаления, как гиперемия конъюнктивы, век, хемоз, отеки век. Патогенез «липогенного» варианта неизвестен. Преобладание процессов адипогенеза предполагает участие иных механизмов аутоиммунного процесса. Ключевую роль в опосредовании глюкокортикоидных реакций в жировой ткани и воспалении играет 11ß-гидроксистероиддегидрогеназа 1 типа (11ß-HSD1), которая повышает биодоступность кортизола в жировых клетках. Индукция активности и экспрессии 11ß-HSD1 воспалительными цитокинами может усиливать дифференцировку орбитальных адипоцитов (адипогенез) и ограничивать пролиферацию других клеток при ЭОП. Предполагается, что $11 \beta$-HSD1 может играть определенную роль в регуляции местной орбитальной воспалительной реакции [7].

\section{Какие же изменения в ткани орбиты}

\section{развиваются при гиперкортицизме?}

Патогенез экзофтальма при гиперкортицизме никак не связан с аутоиммунным воспалением ретробульбарной клетчатки и глазодвигательных мышц, как при болезни Грейвса. Первый случай экзофтальма при гиперкортицизме был упомянут американским нейрохирургом Harvey Williams Cushing в 1932 г. (4 пациента из 12) [8]. В 1987 и 1994 гг. также были описаны клинические случаи синдрома Кушинга, сопровождаемые тяжелым экзофтальмом, требующим оперативного лечения [9]. Далее, в 1996 г., W. Kelly в своих исследованиях изучил группу пациентов с синдромом Кушинга, у 18 из которых отмечались внешние изменения, типичные для офтальмопатии Грейвса. При этом экзофтальм чаще наблюдался у больных с активным синдромом Кушинга (45\% по сравнению с 21\% при ятрогенном генезе заболевания и 20\% больных, получающих терапию) [10].
Как уже говорилось ранее, генез орбитопатии при гиперкортицизме отличается от такового при аутоиммунных заболеваниях щитовидной железы. Чтобы разобраться в истинной причине экзофтальма, следует вспомнить о влиянии гормона надпочечников на организм. Кортизол стимулирует распад белка, что ведет к атрофии мышечной ткани, в том числе с развитием миопатии глазодвигательных мышц. Причина ожирения активный липогенез в одних местах (лицо, туловище) и липолиз в других (конечности) [11]. Под влиянием кортизола отмечается увеличение жировой клетчатки орбит (гормон стимулирует пролиферацию преадипоцитов), что в дальнейшем наравне с гиперпродукцией водянистой влаги приводит к повышению внутриглазного давления. Именно повышенный липогенез в орбитальной области - основная причина экзофтальма при гиперкортицизме. Кроме того, возможно сочетание гиперкортицизма и болезни щитовидной железы (тиреотоксикоз, гипотиреоз). В последнем случае причина орбитопатии - аутоиммунная патология.

Внутриклеточные уровни кортизола регулируются

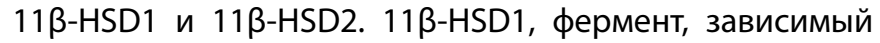
от НАДФ, определяется во многих тканях, в том числе в печени и жировой ткани, и действует преимущественно как редуктаза, генерируя кортизол in vivo. 11ß-HSD2, высокоактивная в почках и толстой кишке, инактивирует кортизол, защищая минералокортикоидный рецептор (MR) от активации [11].

$11 \beta$-HSD1 расположена на люминальной стороне эндоплазматического ретикулума (ЭР), а ее N-концевая часть вставлена в мембрану ЭР [14]. Система, состоящая из транспортера глюкозы-6-фосфата (G6PT) и дегидрогеназа-гексоза-6-фосфата (H6PDH), имеет решающее значение для транспорта G6P к ферменту H6PDH. G6P связывается с H6PDH, в результате чего образуется 6-фосфо-глюконалактон (6PGL), а внутри просвета ER генерируется NADPH. Полученный NADPH используется $11 \beta$-HSD1 для восстановления 11-DHC (B) до кортизола (A). Кортизол взаимодействует со своим рецептором (GR/MR), что не только активирует факторы и гены, отвечающие за адипогенез, но и увеличивает экспрессию $11 \beta$-HSD1 (рис. 3) [15]. 


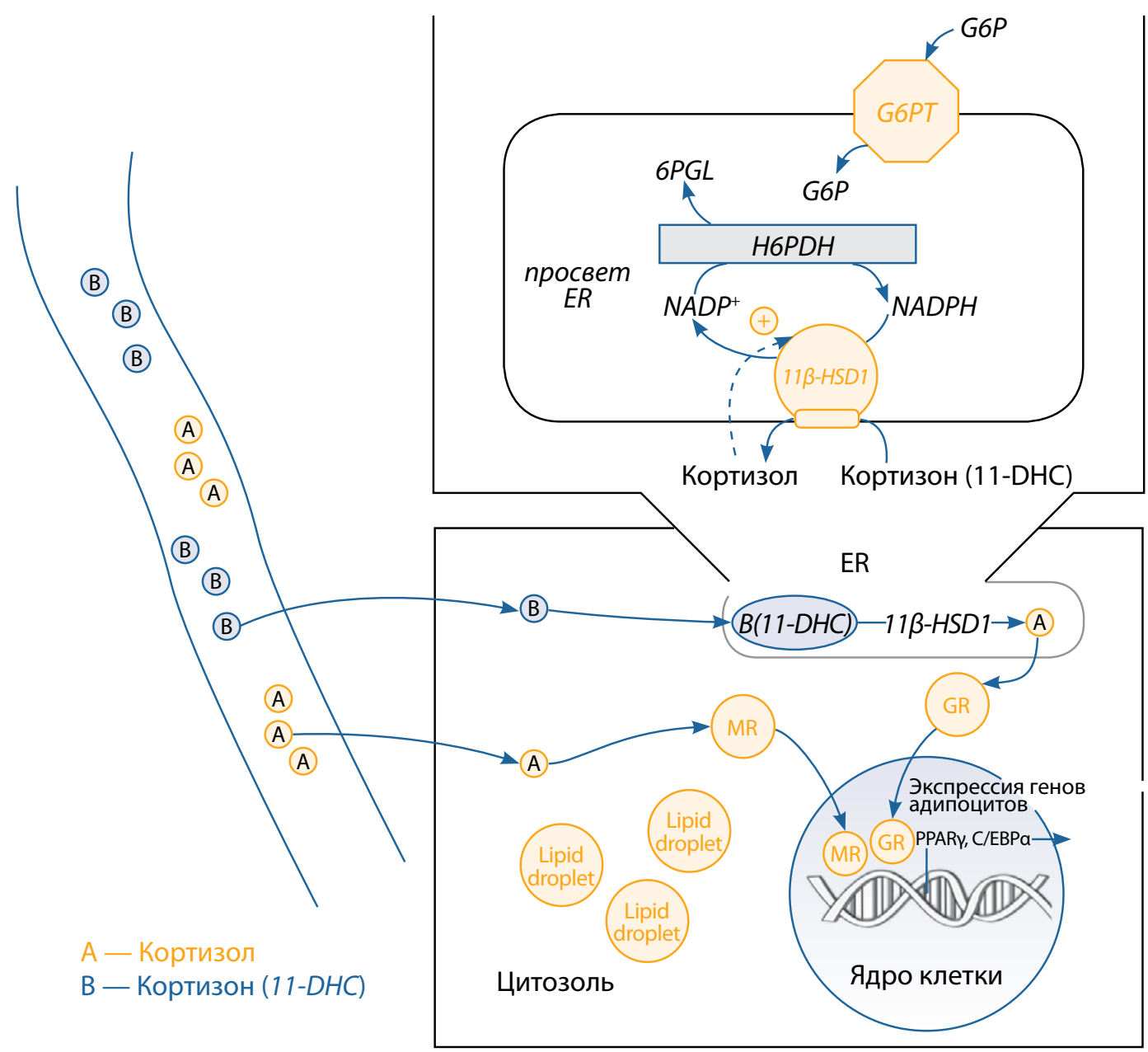

Рисунок 3. Роль кортизола в адипогенезе.

Расположение 11ß-гидроксистероидной дегидрогеназы 1-го типа (11ß-HSD1) в ЭР и ее связь с H6РDH. При синдроме Кушинга цитозольный рецептор минералокортикоидов (MR) активируется именно кортизолом. Большая часть (90\%) циркулирующего кортизола (А) связана с кортикостероидсвязывающим белком (CBG), около 4-5\% - с альбумином, оставшаяся часть гормона свободно диффундирует в адипоцит [12]. Кортизон (B) не так сильно связан с CBG и также диффундирует в клетку, где восстанавливается $11 \beta$-гидроксистероидной дегидрогеназой I (11 $\beta$-HSD1) до A, увеличивая внутриклеточную концентрацию А. Кортизол (A) связывается C MR и глюкокортикоидным рецептором (GR). Эти рецепторы, связавшись с лигандом, транслоцируются в ядро, где стимулируют транскрипцию генов, отвечающих за дифференцировку преадипоцитов в адипоциты и накопление липидных капель (lipid droplet). Из-за низкой активности 11ß-HSD2 - фермента, инактивирующего эндогенный кортизол в клетках, большая часть наблюдаемого эффекта кортизола обусловлена его влиянием на MR, а не на GR [13]. При этом роль GR до конца не известна.

\section{МОЛЕКУЛЯРНЫЕ МЕХАНИЗМЫ АДИПОГЕНЕЗА. РОЛЬ ГИПЕРКОРТИЗОЛЕМИИ В РАЗВИТИИ ОЖИРЕНИЯ}

Исследование плюрипотентных стволовых клеток показало, что миобласты, хондроциты, остеобласты и адипоциты возникают из одних и тех же клеток (стромальных). Дифференцировка преадипоцитов в зрелые жировые клетки происходит во время эмбриогенеза и последующей жизни - в частности, в условиях, ведущих к ожирению, как при гиперкортицизме.

Образовавшиеся из мезенхимальных клеток-предшественников преадипоциты морфологически неотличимы от фибробластов, хотя они уже предетерминированы к развитию в адипоциты. При воздействии на них продифференцирующими агентами (например, инсулином и глюкокортикоидами) они дифференцируются в зрелые клетки спустя 4-6 дней [15].

Весь адипогенный процесс можно разделить на две «волны». Первая инициируется адипогенными стимулами (глюкокортикоиды, инсулин, ИФР1, гормон роста и т.д.) и включает в себя такие факторы, как CCAAT-enhancer- binding protein $\beta(\mathrm{C} / \mathrm{EBP} \beta)$ и CCAAT-enhancer-binding protein $\delta$ (C/EBPS), Krüppel-подобные факторы (например, KLF4 и KLF5) и CREB (сAMP response element-binding protein). Известно, что индукция адипогенеза приводит к быстрому фосфорилированию CAMP (cyclic adenosine monophosphate), CREB, C/EBPß через регуляторные элементы CREB внутри промотора гена C/EBP $\beta$. Сначала C/EВРß не обладает ДНК-связывающей активностью. Однако после длительного периода остановки клеточного роста преадипоциты вновь входят в клеточный цикл и завершают два раунда деления (митотическая клональная экспансия) [15]. В этот момент С/ЕВРß приобретает ДНК-связывающую активность, совпадающую с входом клеток в S-фазу $[16,17]$. C/ЕВРß подвергается фосфорилированию (МАР-киназой и/или киназой GSK-3ß), что индуцирует конформационное изменение C/EBP $\beta$, позволяя ему активировать два ключевых адипогенных транскрипционных фактора второй волны - C/EBPa и PPARү (peroxisome proliferator-activated receptor $\gamma$ ) [17]. Главная роль в дифференцировке жировых клеток принадлежит PPARү. PPARү - мощный активатор адипогенеза, 
он не только превращает фибробласты в адипоциты, но и трансдифференцирует коммитированные миобласты и гепатоциты в адипоциты.

Экспериментально подтверждено, что клетки с нокаутом по С/ЕВРа способны дифференцироваться только при эктопической экспрессии PPAR ки с нокаутом по PPARү вообще не способны к дифференцировке $[17,18]$.

Активируясь, PPARү формирует гетеродимер с ретиноидным X-рецептором (RXR) и запускает экспрессию генов, отвечающих за фенотип зрелых жировых клеток. Кроме того, существует положительная петля обратной связи между C/EBPa и PPARy, она необходима для поддержания дифференцировки адипоцитов (рис. 4) [19, 20].

Лигандами PPARү служат синтетические антидиабетические TZD (розиглитазон, пиоглитазон, троглитазон) и некоторые их метаболиты. Также роль лигандов могут выполнять ADD1 (alpha-adducin) и SREBP1 (sterol regulatory element-binding protein 1) [19]. Выявлено, что эктопическая экспрессия ADD1/SREBP1 в фибробластах способствует адипогенезу путем усиления активности PPAR $\gamma$. Другие же исследования свидетельствуют о том, что ADD1/SREBP1 и C/EBP $\beta$ лишь повышают продукцию лигандов PPAR [20]. При этом сам лиганд все еще не известен.

C/ЕВРа отвечает за уровни инсулиновых рецепторов и одного из их первичных субстратов (Insulin receptor substrate-1), а также за пострецепторную передачу сигналов инсулина. Отсутствие этого фактора приводит к резистентности к инсулину in vitro и препятствует образованию белых жировых клеток in vivo [17].

\section{РОЛЬ ИНСУЛИНА, ИФР И ГЛЮКОКОРТИКОИДОВ В РЕГУЛЯЦИИ АДИПОГЕНЕЗА}

Инсулин, связываясь со своим рецептором, запускает внутриклеточный сигнальный каскад, приводящий к активации киназы Akt и фосфорилированию антиадипогенных транскрипционных факторов FOXO1 (forkhead box protein 01) и FOXA2 (forkhead box protein A2), что вызывает их выход из ядра и деактивацию [20]. Также инсулин является индуктором адипогенеза, он запускает фосфорилирование CREB, белка первой волны. ИФР1 (инсулиноподобный фактор роста 1) стимулирует дифференцировку преадипоцитов. Он связывается со своим рецептором и активирует МАР-киназу, запуская процесс фосфорилирования С/ЕВР $\beta$.

Кортизол активирует глюкокортикоидные и минералокортикоидные рецепторы, которые являются ядерными гормональными рецепторами. Он индуцирует C/EBPs и уменьшает экспрессию preadipocyte factor-1 (pref-1) фактора, ингибирующего адипогенез [17]. Недавние исследования определили LMO3 (LIM Domain Only 3) как участок, с которым взаимодействуют глюкокортикоиды. Выявлено, что область промотора LMO3 содержит два полусайта GRE, с которыми связывается кортизол. Механизм, с помощью которого LMO3 регулирует дифференцировку адипоцитов, также известен. LMO3 напрямую ингибирует ERK-опосредованное фосфорилирование PPARg (peroxisome proliferator-activated receptor g) на Ser112 - сайте, блокирующем активацию PPARg-зависимой адипогенной программы [21].

\section{Глюкокортикоиды и бурая жировая ткань}

Считается, что метаболически активная бурая жировая ткань присутствует у взрослых людей и может играть роль в энергетическом гомеостазе и контроле веса. Кортизол способствует дифференцировке преадипоцитов, но подавляет экспрессию разобщающего белка 1 (UCP1) в бурых жировых клетках. Снижая термогенетическую активность UCP1 и увеличивая накопление липидов внутри клеток, кортизол нарушает основную функцию бурой жировой ткани и способствует ее переходу к «белому» фенотипу [22].

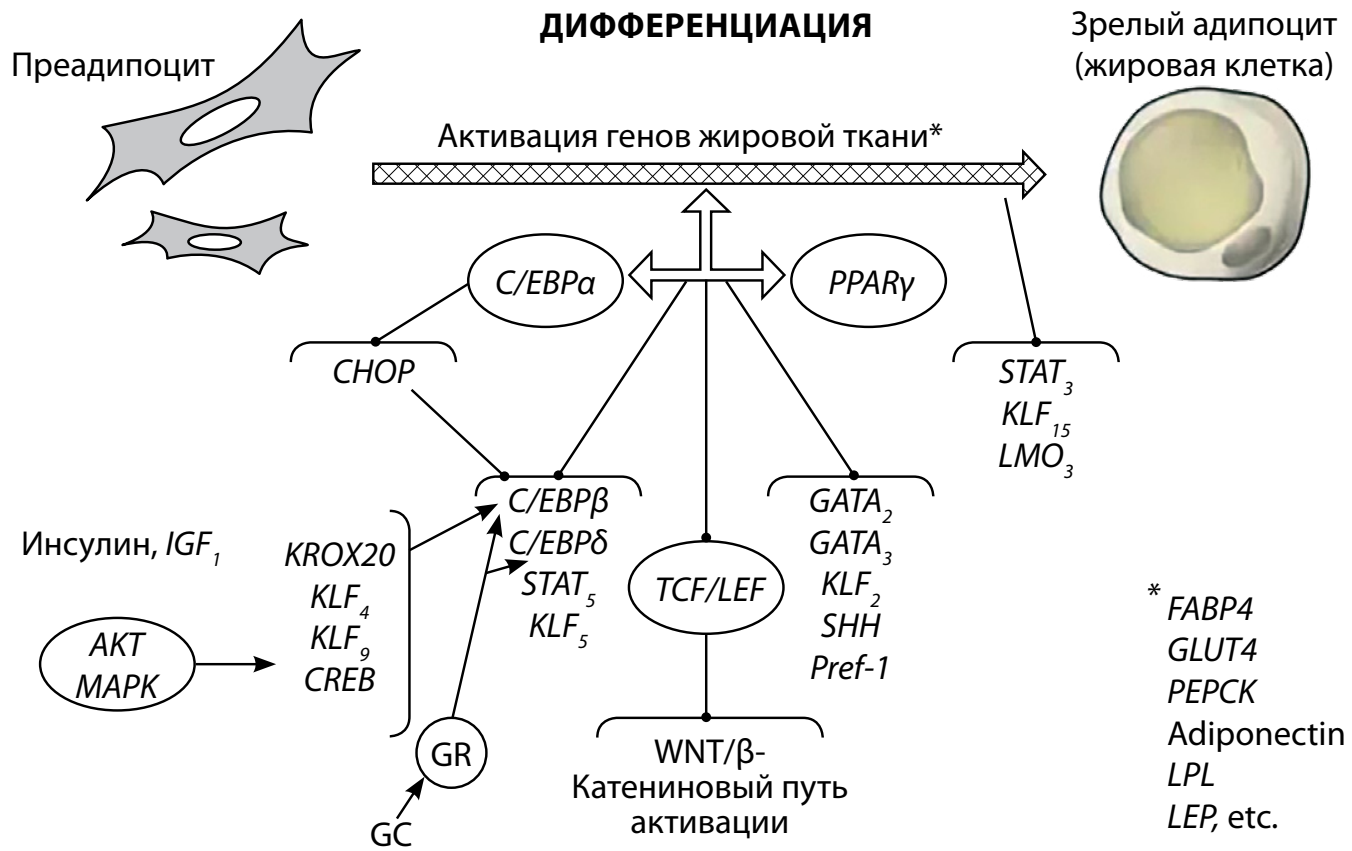

Рисунок 4. Набросок транскрипционного каскада, управляющего дифференцировкой адипоцитов.

Главные адипогенные стимулы включают в себя глюкокортикоиды и инсулин. Действуя синергически с инсулином, глюкокортикостероиды увеличивают экспрессию многочисленных генов, участвующих в отложении жира [11]. Эти гормоны активируют первую волну адипогенных транскрипционных факторов. Хорошо изучены два из них - C/EBP $\beta$ и C/EBPठ. Они индуцируют вторую волну, в которой PРARg и С/ЕВРа играют важную роль $[17,18]$. 


\section{Индукторы и ингибиторы адипогенеза}

Формирование преадипоцитов, а потом и адипоцитов из мезенхимальных стволовых клеток основано на ингибировании других программ развития (хондроцитов, остеоцитов и миоцитов). Транскрипционный фактор 2 (Runx2) и костный морфогенетический белок (КМБ)-2 являются остеогенными факторами. При активации адипоцитарного пути развития под влиянием кортизола и других стимулов они подавляются микроРНК [20]. Известно, что 204 и 30 микроРНК ингибируют Runx2, а 17-5р и 106а - КМБ-2. Хондрогенез контролируется TGF- $\beta$ (transforming growth factor beta), который блокируется miR-21. Экспрессия этой микроРНК увеличивается при ожирении, сахарном диабете 2-го типа, метаболическом синдроме [12]. Проадипогенные микроРНК, такие как miR-148 и 124, ингибируют Wnt1 и Sox9 (SRY-Box Transcription Factor 9), белки, участвующие в хондрогенезе [22]. Проадипогенные miR-143 и -103 увеличиваются при дифференцировке адипоцитов и играют важную роль в накоплении липидов, а ингибирование PPARa miR-519d снижает окисление жирных кислот [18].

Известными антиадипогенными микроРНК являются miR-130 и miR-27 [22]. Они ингибируют PPARy, главный активатор адипогенеза.

Также микроРНК регулируют передачу инсулинового сигнала в адипоцитах. Они могут ослаблять инсулиновую сигнализацию, воздействуя на инсулиновый рецептор, IRS1 и GLUT4. Стабильность инсулинового рецептора частично зависит от белка caveolin-1, являющегося мишенью для проадипогенной miR-103. Таким образом, ингибирование miR-103 повышает стабильность инсулинового рецептора и увеличивает чувствительности к инсулину. IRS1 снижается при помощи микроРНК-139-5 и -144, а поглощение глюкозы через GLUT4 уменьшается при высокой экспрессии микроРНК-93 и -223 [18, 22].

Хоть PPARg и C/EBPa являются неотъемлемой частью транскрипционного каскада, контролирующего адипогенез, другие факторы, такие как signal transducer and activator of transcription 3,5 (STAT3, STAT5) и Kruppel-like factor 15 (KLF15), также регулируют этот процесс (рис. 4). Они были предложены в качестве положительных регуляторов дифференцировки жировых клеток на основании вывода о том, что запуск РНК-интерференции этих факторов ингибирует адипогенез в культуре клеток. Предполагается, что GATA2/3 и KLF2 являются отрицательными регуляторами адипогенеза, так как чрезмерная экспрессия этих факторов ингибирует адипогенез в 3T3-L1, линии клеток, используемой для изучения жировой ткани [15]. Однако большинство этих факторов не было подтверждено в качестве регуляторов адипогенеза в физиологических условиях с использованием животных моделей, и их гены-мишени точно неизвестны.

PPARY и C/EBPa индуцируют экспрессию генов, участвующих в регуляции инсулиновой чувствительности, липогенезе и липолизе. К ним относят ген, отвечающий за экспрессию GLUT4 (известный как SLC2A4), ген ЛПЛ (липопротеинлипазы), AGPAT2 (1-Acylglycerol-3-Phosphate O-Acyltransferase 2), FABP4 (Fatty Acid Binding Protein 4) и гены $A D I P O Q$ и $L E P$, отвечающие за синтез адипонектина и лептина соответственно $[18,19]$. Другие положительные регуляторы адипогенеза включают факторы
EGR2 (ген EGR2), EBF1 (ген EBF1). Для простоты указывается только подмножество транскрипционных факторов и взаимосвязей.

\section{Wnt и ожирение}

Пути Wnt представляют собой группу сигнальных путей трансдукции, которые начинаются с белков, передающих сигналы внутрь клетки. Название Wnt является словом-гибридом и дословно расшифровывается как «сайт интеграции безымянных».

Путь Wht - это древний путь, регулирующий судьбу клеток, их пролиферацию и миграцию, полярность, а также аспекты органогенеза в процессе эмбрионального развития. Сигналы Wnt активны во многих случаях, участвуя сначала на ранних стадиях развития, а затем в процессе роста и самообновления различных тканей. Белки Wnt связываются со специфическими рецепторами (рецепторами семейств Frizzled и LRP) на поверхности клетки. Далее, в каноническом пути, сигнал передается на ß-катенин [4]. При активации этого пути накапливающийся свободный $\beta$-катенин проникает в ядро, где он связывается c LEF/TCF (lymphoid enhancer binding factor/T-cell specific transcription factor) и запускает транскрипцию генов-мишеней Wnt [23, 24]. Wnt/ $\beta$-catenin pathway ингибирует адипогенез (блокируя PPARg и CEBPA). И наоборот, нарушение сигнализации Wnt приводит к спонтанной дифференцировке адипоцитов. Кроме того, активация Wnt/ $\beta$-catenin pathway способствует коммитированию MSC (multipotent stem cells) с их развитием в миоциты и остеоциты, тем самым подавляя адипоцитарную линию и ингибируя терминальные стадии дифференцировки адипоцитов [24].

Различные исследования демонстрируют важность сигнального пути Wnt в развитии адипоцитов как in vitro, так и in vivo, а также то, что подавление этого пути необходимо для продолжения дифференцировки жировых клеток. Похожим образом сигнальный путь Sonic hedgehog (SHH) ингибирует адипогенез, хотя конкретные механизмы мало изучены [19]. Предполагается, что рецепторы, которые инициируют пути сигнализации Wht и $\mathrm{HH}$, находятся на первичных ресничках, сенсорной органелле, временно присутствующих на дифференцирующихся адипоцитах. Повреждение ресничек в процессе адипогенеза приводит к повышенной экспрессии PPARү [18].

SFRP1 также участвует в регуляции адипогенеза у человека. Белок, кодируемый этим геном, функционирует как модулятор сигнализации Wnt (является антагонистом) и регулирует рост и дифференцировку клеток, снижая внутриклеточный уровень бета-катенина. Анализируя экспрессию генов, контролирующих адипогенез, Kumar и соавт. определили SFRP1 как один из наиболее высокорегулируемых транскриптов в жировых клетках орбит у пациентов с тяжелой ЭОП. Рекомбинантный SFRP1 in vitro усиливал дифференцировку преадипоцитов орбит при орбитопатии Грейвса, что указывало на его патогенетическую роль в развитии данной патологии [24].

\section{Другие регуляторы адипогенеза}

Транскрипционные коактиваторы, такие как белки-медиаторы и SRC, функционируют как адаптеры, которые соединяют транскрипционные факторы с Pol II или гистонацетилтрансферазой для активации экспрессии генов. Показано, что для адипогенеза в культуре клеток 
требуется несколько медиаторов, в том числе Med1, Med14 и Med23, а также несколько компонентов семейства SRC, в том числе SRC-2 и SRC-3 [16]. Однако роль этих коактиваторов в адипогенезе до конца не определена.

Важно отметить, что данные, полученные in vitro c использованием экспериментальных моделей, следует интерпретировать с осторожностью. К примеру, ряд исследований исключают EGR2, KLF4 из списка адипогенных факторов in vivo, объясняя это тем, что их положительное влияние на адипогенез было подтверждено лишь in vitro на 3Т3-линии $[16,23]$.

\section{ДИФФЕРЕНЦИАЛЬНАЯ ДИАГНОСТИКА ЭКЗОФТАЛЬМА КАК ОДНОГО ИЗ СИМПТОМОВ ЭНДОКРИННОЙ ПАТОЛОГИИ}

Интересные наблюдения были сделаны Robert G. Peyster и соавт. в области дифференциальной диагностики экзофтальма по данным КТ. Была изучена группа пациентов с проптозом, у части пациентов диагностировали орбитопатию Грейвса, часть - имели болезнь/синдром Кушинга, а остальные - проптоз вследствие ожирения (без эндокринопатии). Проводились измерения орбитального объема и процентного объема орбитального жира с помощью КТ у данных пациентов и в контрольной группе из 16 человек без пропозиции. Кроме того, проводилось измерение толщины подкожножировой клетчатки головы на уровне волосяных луковиц. Больший объем орбитального жира был обнаружен в группе пациентов с проптозом по сравнению с контрольной группой. Отмечалось, что степень проптоза прямо пропорциональна увеличению орбитальной жировой клетчатки. Орбитальный объем существенно не изменялся у пациентов с проптозом по сравнению с контрольной группой. Толщина жирового слоя волосистой части головы на уровне луковиц была значительно больше у пациентов с ожирением и синдромом/болезнью Кушинга, чем у контрольной группы и у пациентов с болезнью Грейвса (в последнем случае изменения незначительны). Результаты данного исследования подтвердили, что причина проптоза у разных групп пациентов - в увеличении орбитального жира. Другие параметры, использовавшиеся в исследовании, могут помочь в дифференциальной диагностике в случае, если нет других клинических данных. При этом важно помнить, что пациенты с болезнью Грейвса также могут страдать ожирением, а пациенты с ожирением при необходимости (если есть другие симптомы) могут быть обследованы на наличие эндокринной патологии [25].

Возникает еще один вопрос: почему ожирение при синдроме Кушинга встречается почти всегда, а экзофтальм - нет? Скорее всего, причина в разном строении адипоцитов орбит и клеток подкожножировой клетчатки. Существует мнение, что орбитальный жир имеет ряд характеристик бурой жировой ткани [26].

По данным Onyimba C. и соавт., адипоциты орбитального жира мельче и менее дифференцированы, чем аналогичные клетки подкожной жировой клетчатки. Кроме того, уровень экспрессии 11ß-HSD1 у них ниже, а GR, H6PDH - выше [27]. Большая плотность глюкокортикоидных и минералокортикоидных рецепторов, высокий уровень экспрессии Н6PDH и повышение активности липопротеиновой липазы объясняют увеличение орбитальной жировой клетчатки при гиперкортицизме, а отличное количество $11 \beta$-HSD1, фермента, способствующего превращению кортизона в кортизол, может быть причиной не столь стремительных изменений.

\section{ДРУГИЕ ОРБИТАЛЬНЫЕ ПРОЯВЛЕНИЯ ГИПЕРКОРТИЦИЗМА}

Кроме экзофтальма у ряда пациентов с длительным течением заболевания возможно развитие глаукомы (5\% случаев) с характерными нарушениями зрительных функций и состоянием глазного дна (глаукоматозная экскавация диска зрительного нерва). Особенности глаукомы при болезни Иценко-Кушинга - симметричность показателей гидродинамики и дистрофических изменений в тканях глаза, сохранность зрительных функций длительное время. Ниже описан клинический случай, в котором орбитальные проявления и некоторые другие неспецифические симптомы послужили поводом для обследования пациента на наличие синдрома Кушинга.

Глазная гипертензия может быть симптомом болезни Кушинга, вызванным длительным воздействием высокого уровня кортизола. В 2019 г. в Великобритании группа исследователей представила случай 35-летнего мужчины с высоким внутриглазным давлением, обнаруженным во время планового обследования. Пациенту, имеющему нормальное зрение, был поставлен диагноз «глазная гипертензия с риском глаукомы». Из анамнеза известно, что пациент страдает ожирением в течение 5 лет. Кроме того, за последние годы у него изменились черты лица и отмечалось повышенное артериальное давление. Также мужчина жаловался на общую слабость и беспричинное образование синяков. Врач заподозрил болезнь Кушинга и направил пациента к эндокринологу. Анализы крови показали, что у пациента повышен уровень сахара до значений, характерных для сахарного диабета. Кроме того, отмечались высокий уровень холестерина в крови и повышенный уровень кортизола в моче. В ходе дальнейшей диагностики врачи обнаружили повышенный уровень АКТГ. При МРТ гипофиза была найдена микроаденома, и диагноз был подтвержден. На период до проведения транссфеноидальной аденомэктомии пациенту был выписан метирапон. Сообщалось также о трех других случаях болезни Кушинга у молодых пациентов, где в результате гиперкортизолемии развивалось повышение внутриглазного давления с риском возникновения глаукомы [28].

\section{Катаракта как следствие синдрома Кушинга}

В 1993 г. Evridiki A. Bouzas и соавт. решили рассмотреть группу пациентов с синдромом Кушинга на предмет орбитальной патологии. Дело в том, что известным осложнением длительной глюкокортикоидной терапии является задняя подкапсульная катаракта. Целью этого исследования было изучение влияния хронического эндогенного гиперкортизолизма на хрусталик человека. Были изучены 60 пациентов (от 8 до 67 лет, 46 женщин, 14 мужчин) с подтвержденным эндогенным синдромом Кушинга. Только у двух из 60 пациентов (3,3\%) была выявлена задняя подкапсульная катаракта. Такая низкая распространенность контрастирует с высокой распространенностью, обусловленной глюкокортикоидной терапией с эквивалентной дозой глюкокортикоидов. На основании этого можно сделать вывод, что задняя 
подкапсульная катаракта является редким осложнением эндогенного гиперкортизолизма по сравнению с экзогенным синдромом Кушинга. Поскольку общее воздействие эндогенных глюкокортикоидов было не ниже, чем при экзогенной глюкокортикоидной терапии, потенциальным объяснением этой разницы могут быть фармакокинетические различия синтетических глюкокортикоидов и кортизола человека [29].

\section{Узелки Лиша и центральная серозная \\ хориоретинопатия у пациентов \\ с гиперкортицизмом}

Два других уникальных офтальмологических признака были также обнаружены у пациентов с синдромом Кушинга. Узелки Лиша, специфические для нейрофиброматоза 1 типа, редко встречаются при других заболеваниях. Они являются меланоцитарной гамартомой радужной оболочки глаза. Эти желто-коричневые куполообразные возвышения, выступающие над поверхностью радужки, были найдены у 2 из 14 пациентов с эндогенным гиперкортицизмом. Предполагается, что основной механизм, приводящий к их появлению в радужной оболочке глаза, похож на механизм возникновения кортикотрофных аденоматозных изменений в гипофизе. В тех же исследованиях сообщалось о наличии центральной серозной хориоретинопатии (ЦСХ) у 3 из 60 пациентов с синдромом Кушинга. ЦСХ представляет собой заболевание, характеризующиеся накоплением субретинальной жидкости на заднем полюсе глазного дна (за сетчаткой). В результате этого у пациентов возникают зоны отслоения сетчатки и ухудшается зрение. Известно, что мужчины страдают этим заболеванием чаще, чем женщины, но хориоретинопатии довольно редко встречаются даже у пациентов с синдромом Кушинга, у которых распространенность этого заболевания, вероятно, составляет менее 5\%. Хориоретинопатия связана с повышенным уровнем эндогенных или экзогенных глюкокортикоидов. Ниже представлены случаи ЦСХ у пациентов с синдромом Кушинга.

40-летний мужчина с нарушением зрения на фоне центральной серозной ретинопатии был направлен офтальмологом для диагностики гипоталамо-гипофизарной патологии. МРТ гипофиза выявила наличие крупной макроаденомы гипофиза с компрессией зрительного нерва на уровне хиазмы и с вовлечением кавернозного синуса. Дальнейшая диагностика подтвердила болезнь Кушинга с АКТГ в 9:00 утра более 1000. Пациенту была произведена транссфеноидальная декомпрессионная операция, в результате которой острота зрения улучшилась, а АКТГ снизился до 360 [30].

В следующем случае 39-летний мужчина с двусторонней хориоретинопатией (с 1999 г.) провел собственный поиск в интернете и нашел связь между ЦСХ и синдромом Кушинга. На визите у терапевта пациент стал настаивать на том, чтобы его проверили на наличие синдрома Кушинга. Несмотря на то что у него не было явных признаков заболевания, диагностика эндокринной системы, включая селективный забор крови из нижних каменистых синусов и МРТ, подтвердила наличие аденомы гипофиза. Впоследствии пациент был направлен на транссфеноидальную аденомэктомию [30].
Кроме того, пациенты с ЦСХ были описаны в работах Suri N. Apра и Cameron Clarke. В обоих случаях был диагностирован АКТГ-зависимый синдром Кушинга (микрои макроаденома соответственно) [31, 32].

Опираясь на приведенные исследования, можно сделать вывод, что основные глазные проявления гиперкортицизма включают повышение внутриглазного давления и экзофтальм, причем последний развивается из-за активного адипогенеза в области орбит. Катаракта возникает как осложнение длительной терапии кортикостероидами и в основном наблюдается при ятрогенном синдроме Кушинга [33]. ЦСХ может быть следствием длительной гиперкортизолемии, поэтому пациенты с данным заболеванием должны быть опрошены на предмет наличия у них признаков синдрома Кушинга. Кроме того, пациенты с синдромом Кушинга могут страдать диабетом, а изменения в сетчатке могут быть вызваны повышенным уровнем глюкозы в крови.

В настоящее время не разработана единая схема оценки тяжести орбитальных изменений при гиперкортицизме. Но это и не является первоначальной задачей. Дело в том, что изменения мягких тканей глазницы при гиперкортицизме характеризуются относительно скудными клиническими проявлениями. Нередко пациенты не предъявляют жалобы, при этом экзофтальм выражен незначительно $[8,9]$. Кроме того, лечение основного заболевания и нормализация уровня кортизола приводят к снижению выраженности экзофтальма и исчезновению глазных симптомов [9].

\section{ЗАКЛЮЧЕНИЕ}

Причина развития экзофтальма при гиперкортицизме еще мало изучена. Это объясняется тем, что экзофтальм относительно редко встречается как отдельный симптом при синдроме Кушинга. Несмотря на это, все данные свидетельствуют о связи экзофтальма с повышенным уровнем кортизола в организме (особенно локально) и активностью ферментов, участвующих в реализации его эффектов.

\section{ДОПОЛНИТЕЛЬНАЯ ИНФОРМАЦИЯ}

Источник финансирования. Данная работа выполнена при финансовой поддержке Российского научного фонда (грант РНФ №17-75-30035).

Конфликт интересов. Авторы декларируют отсутствие явных и потенциальных конфликтов интересов, связанных с публикацией настоящей статьи.

Участие авторов: Корчагина М.О. - набор и изучение материала, написание статьи, создание схематических изображений; Трухин А.А. - редактирование статьи, оцифровка схематических изображений; Свириденко Н.Ю. - набор материала, написание статьи.

Все авторы одобрили финальную версию статьи перед публикацией, выразили согласие нести ответственность за все аспекты работы, подразумевающую надлежащее изучение и решение вопросов, связанных с точностью или добросовестностью любой части работы

Благодарности. Мельниченко Г.А. - за подбор темы для обзора, наставничество, редактирование статьи; Пигаровой Е.А. - за руководство, подачу идей для раскрытия темы, помощь на первоначальных этапах написания статьи; Рыбиной Е.С. - за работу над английской частью рукописи. 


\section{СПИСОК ЛИТЕРАТУРЫ | REFERENCES}

1. Российская ассоциация эндокринологов и нейрохирургов. Болезнь Иченко-Кушинга. Клинические рекомендации. - М.: Министерство здравоохранения Российской Федерации; 2016. [Rossijskaja associacija endokrinologov i nejrohirurgov. Bolezn'lcenko-Kushinga. Klinicheskie rekomendacii. Moscow: Ministerstvo zdravoohranenija Rossijskoj Federacii; 2016. (In Russ.)].

2. Мельниченко Г.А., Дедов И.И., Белая Ж.Е., и др. Болезнь Иценко-Кушинга: клиника, диагностика, дифференциальная диагностика, методы лечения // Проблемы эндокринологии. 2015. - T. 61. — №2. - C. 55-77. [Melnichenko GA, Dedov II, Belaya ZE, et al. Cushing's disease: the clinical features, diagnostics, differential diagnostics, and methods of treatment. Problems of Endocrinology. 2015;61(2):55-77. (In Russ.)]. doi: https://doi.org/10.14341/probl201561255-77

3. Findling JW, Raff H. Screening and diagnosis of Cushing's syndrome. Endocrinol Metab Clin North Am. 2005;34(2):385-x. doi: https://doi.org/10.1016/j.ecl.2005.02.001

4. Clinicaltrials [Internet]: U.S. National Library of Medicine; 2020 Hypercorticism, Russia. Available from: https://clinicaltrials.gov/ ct2/results?cond=Hypercorticism\&term $=\&$ cntry $=$ RU\&state $=\&$ city $=\&$ dist $=\&$ Search $=$ Search

5. Giugni AS, Mani S, Kannan S, Hatipoglu B. Exophthalmos: A Forgotten Clinical Sign of Cushing's Syndrome. Case Rep Endocrinol. 2013;2013(2):1-3. doi: https://doi.org/10.1155/2013/205208

6. Дедов И.И., Мельниченко Г.А., Свириденко Н.Ю., и др. Федеральные клинические рекомендации по диагностике и лечению эндокринной офтальмопатии при аутоиммунной патологии щитовидной железы // Проблемы эндокринологии. - 2015. - Т. 61. - №1. - С. 61-74. [Dedov II, Melnichenko GA, Sviridenko NY, et al. Federal clinical recommendations on diagnostics and treatment of endocrine ophthalmopathy associated with autoimmune thyroid pathology. Problems of Endocrinology. 2015;61(1):61-74. (In Russ.)]. doi: https://doi.org/10.14341/probl201561161-74

7. Durrani O.M, Onyimba CU, Bujalska IJ, et al. Thyroid Associated Ophthalmopathy - Cushing's Disease of the Orbit? Invest. Ophthalmol. Vis. Sci. 2007;48(13):3573.

8. Cushing $\mathrm{H}$. The basophil adenomas of the pituitary body and their clinical manifestation. Bulletin Johns Hopkins Hospital. 1932;50:173-195.

9. Giugni AS, Mani S, Kannan S, et al. Exophthalmos: A Forgotten Clinical Sign of Cushing's Syndrome. Case Rep Endocrinol. 2013;2013:205-208. doi: https://doi.org/10.1155/2013/205208

10. Kelly W. Exophthalmos in Cushing's syndrome. Clin Endocrinol (Oxf). 1996;45(2):167-170. doi: https://doi.org/10.1046/j.1365-2265.1996.d01-1559.x

11. Lee M-J, Pramyothin P, Karastergiou K, Fried SK. Deconstructing the roles of glucocorticoids in adipose tissue biology and the development of central obesity. Biochim Biophys Acta - Mol Basis Dis. 2014;1842(3):473-481. doi: https://doi.org/10.1016/j.bbadis.2013.05.029

12. Paredes S, Ribeiro L. Cortisol: the villain in Metabolic Syndrome? Rev Assoc Med Bras. 2014;60(1):84-92. doi: https://doi.org/10.1590/1806-9282.60.01.017

13. Gomez-Sanchez CE. What is the role of the adipocyte mineralocorticoid receptor in the metabolic syndrome? Hypertension. 2015;66(1):17-19. doi: https://doi.org/10.1161/HYPERTENSIONAHA.115.05148

14. Артемова Е.В. Особенности синтеза, активации и дезактивации глюкокортикоидов. Биологическая роль кортизола в метаболических нарушениях // Ожирение и метаболизм. - 2017. - Т. 14. — №2. - 48-52. [Artemova EV. Synthesis, activation and deactivation of glucocorticoids. The biological role of cortisol in metabolic disorders. Obesity and metabolism. 2017;14(2):48-52. (In Russ.)]. doi: https://doi.org/10.14341/omet2017248-52
15. Morgan S, McCabe E, Gathercole L, et al. 11-HSD1 is the major regulator of the tissue-specific effects of circulating glucocorticoid excess. Proceedings of the National Academy of Sciences. 2014;111(24):E2482-E2491. doi: https://doi.org/10.1073/pnas.1323681111

16. Lee J-E, Schmidt H, Lai B, Ge K. Transcriptional and Epigenomic Regulation of Adipogenesis. Mol Cell Biol. 2019;39(11) doi: https://doi.org/10.1128/MCB.00601-18

17. Rosen $E$, MacDougald $O$. Adipocyte differentiation from the inside out. Nature Reviews Molecular Cell Biology. 2006;7(12):885-896. doi: https://doi.org/10.1038/nrm2066

18. Lowe C, O'Rahilly S, Rochford J. Adipogenesis at a glance. J Cell Sci. 2011;124(21):3726-3726. doi: https://doi.org/10.1242/jcs.101741

19. Moseti D, Regassa A, Kim W-K. Molecular Regulation of Adipogenesis and Potential Anti-Adipogenic Bioactive Molecules. Int J Mol Sci. 2016;17(1):124. doi: https://doi.org/10.3390/ijms17010124

20. Егоров А.Д., Пеньков Д.Н., Ткачук В.А. Молекулярные и клеточные механизмы адипогенеза // Сахарный диабеm. - 2015. - T. 18. - №2. - C. 12-19. [Egorov AD, Penkov DN, Tkachuk VA. Molecular and cellular mechanisms of adipogenesis. Diabetes mellitus. 2015;18(2):12-19. (In Russ.)]. doi: https://doi.org/10.14341/DM2015212-19

21. Galitzky J, Bouloumié A. Human Visceral-Fat-Specific Glucocorticoid Tuning of Adipogenesis. Cell Metab. 2013;18(1):3-5. doi: https://doi.org/10.1016/j.cmet.2013.06.008

22. Luong Q, Huang J, Lee KY. Deciphering White Adipose Tissue Heterogeneity. Biology (Basel). 2019;8(2):23. doi: https://doi.org/10.3390/biology8020023

23. Chen J, Yang Y, Li S, et al. E2F1 Regulates Adipocyte Differentiation and Adipogenesis by Activating ICAT. Cells. 2020;9(4):1024. doi: https://doi.org/10.3390/cells9041024

24. Christodoulides C, Lagathu C, Sethi J, et al. Adipogenesis and WNT signalling. Trends in Endocrinology \& Metabolism. 2009;20(1):16-24 doi: https://doi.org/10.1016/j.tem.2008.09.002

25. Peyster R, Ginsberg F, Silber J, et al, Exophthalmos caused by excessive fat: CT volumetric analysis and differential diagnosis. American Journal of Roentgenology. 1986;146(3):459-464.

26. Борзенок С.А., Гущина М.Б., Афанасьева Д.С., и др. Орбитальная жировая клетчатка - новый ресурс для трансплантологии // Вестник трансплантологии и искусственных органов. 2015. - T. 17. — №4. - C. 118-123. [Borzenok SA, Gushchina MB, Afanasyeva DS, et al. Orbital adipose tissue is a new resource for transplantology. Russian Journal of Transplantology and Artificial Organs. 2015;17(4):118-123. (In Russ.)]. doi: https://doi.org/10.15825/1995-1191-2015-4-118-123

27. Onyimba C, Bujalska I, Durrani O, et al. Glucocorticoid metabolic pathways in human orbital adipose tissue: a comparison with subcutaneous and omental depots. Endocrine Abstracts. 2007; 13:115

28. Habib SN, Lin Z, Puvanachandra N. Ocular hypertension secondary to high endogenous steroid load in Cushing's disease. BMJ Case Rep. 2019;12(1):bcr-2018-226738. doi: https://doi.org/10.1136/bcr-2018-226738

29. Bouzas EA, Mastorakos G, Friedman TC et al. Posterior subcapsular cataract in endogenous Cushing syndrome: an uncommon manifestation. Invest Ophthalmol Vis Sci. 1993;34(13):3497-3500.

30. Ibrahim IM, Al-Bermani A, James RA. Ophthalmic presentations of Cushing's syndrome. Presented at Society for Endocrinology BES, Glasgow, UK. Endocrine Abstracts 2006;11:79.

31. Appa S. Subclinical hypercortisolism in central serous chorioretinopathy. Retin Cases Brief Rep. 2014;8(4):310-313. doi: https://doi.org/10.1097/icb.0000000000000036

32. Clarke C, Smith SV, Lee AG. A rare association: Cushing disease and central serous chorioretinopathy. Can J Ophthalmol. 2017;52(2):e77-e79. doi: https://doi.org/10.1016/j.jcjo.2016.09.002

33. Jacob J, Chopra R, Chander A. The eye as a window to rare endocrine disorders. Indian J Endocrinol Metab. 2012;16(3):331. doi: https://doi.org/10.4103/2230-8210.95659 


\section{ИНФОРМАЦИЯ ОБ АВТОРАХ [AUTHORS INFO]}

*Корчагина Мария Олеговна [Maria O. Korchagina]; адрес: Россия, 117186, Москва, ул. Нагорная, д. 17, к. 3 [Nagornaya street 17/3, 117186, Moscow, Russia]; телефон: 89153375125; ORCID: 0000-0002-6954-1126; eLibrary SPIN:7834-5652; e-mail: kmylavender@gmail.com

Трухин Алексей Андреевич [Alexey A. Trukhin]; ORCID: 0000-0001-5592-4727; eLibrary SPIN: 4398-9536; e-mail: Alexey.trukhin12@gmail.com

Свириденко Наталья Юрьевна, д.м.н., професcop [Natalya Yu. Sviridenko, MD, PhD, Professor];

ORCID: http://orcid.org/0000-0002-8538-5354; eLibrary SPIN: 5889-6484; e-mail: natsvir@inbox.ru

\section{ЦИТИРОВАТЬ}

Корчагина М.О., Трухин А.А., Свириденко Н.Ю. Орбитальные проявления гиперкортицизма // Клиническая и экспериментальная тиреоидология. - 2020. - Т. 16. - №4. - С. 4-13. doi: https://doi.org/10.14341/ket12699

Рукопись получена: 01.02.2021. Одобрена к публикации: 07.04.2021

\section{TO CITE THIS ARTICLE}

Korchagina MO, Trukhin AA, Sviridenko NYu. Orbital manifestations of hypercorticism. Clinical and experimental thyroidology. 2020;16(4):4-13. doi: https://doi.org/10.14341/ket12699

Received: 01.02.2021. Accepted: 07.04.2021 